\title{
CÁNCER TIROIDEO DIFERENCIADO, MANEJO QUIRÚRGICO
}

\section{Differentiated thyroid cancer, surgical management}

Fecha de recibido: 09-09-2010 • Fecha de aprobación: 22-10-2010

Resumen. El cáncer tiroideo es la neoplasia endocrina más frecuente, sin embargo solo causa el 1\% de todos los cánceres humanos. De acuerdo a su histología y comportamiento biológico son divididos en bien diferenciados y no diferenciados. Las opciones de manejo quirúrgico del cáncer bien diferenciado incluyen tiroidectomía casi total, total, subtotal y hemitiroidectomía más istmectomía. La extensión y tipo de cirugía continúan siendo un área de controversia. Las complicaciones asociadas a la cirugía son directamente proporcionales a la extensión de la cirugía e inversamente a la experiencia del cirujano, incluyendo, sangrado, infección del sitio operatorio, seroma, lesión nerviosa, hipoparatiroidismo, hemorragia postquirúrgica y obstrucción de la vía aérea. Este artículo revisa las opciones terapéuticas en el manejo quirúrgico del cáncer de tiroides.

Palabras clave: neoplasias de tiroides, glándula tiroides, tiroidectomía, complicaciones postoperatorias.

Abstract. Thyroid cancer is the most frequent endocrine neoplasia; however, it only causes $1 \%$ of all human cancers. According to its histology and biological behavior they are divided into well-differentiated and non-differentiated. Surgical management options of well-differentiated cancer include almost total, total, subtotal thyroidectomy and hemithyroidectomy plus isthmectomy.

Extension and kind of surgery is still under controversy. Complications related to surgery are directly proportional to the surgery extension and inversely proportional to the surgeon's experience, including bleeding, operation site infection, seroma, nerve injury, hyperparathyroidism, post surgical bleeding and airway obstruction. This article reviews the therapeutic options in surgical management of thyroid cancer.

Key words: thyroid neoplasms, thyroid gland, thyroidectomy, postoperative complications.

\section{INTRODUCCIÓN}

El cáncer tiroideo es la neoplasia endocrina más frecuente, sin embargo solo representa menos del $1 \%$ de todos los tumores en el hombre ${ }^{(1)}$. Son derivados de las células foliculares tiroideas y se clasifican en cáncer bien diferenciado (CTD) y no diferenciado. Los CTD incluyen cáncer papilar (CTP), que es el más común, cáncer folicular, cáncer de células de Hurtle y tumores mixtos que contienen tanto células papilares como foliculares. El cáncer no diferenciados o pobremente diferenciado, se refiere a cáncer insular o anaplásico (CA). El cáncer medular se deriva de las células parafoliculares y posee un comportamiento biológico diferente.

Tumores poco frecuentes como linfoma, sarcoma, carcinoma de células escamosas y tumores metastásicos principalmente derivados de neoplasias epiteliales (mama, colon, riñón, ovario y pulmón) se pueden desarrollar en la glándula tiroides ${ }^{(2)}$.

* Cirujano de cabeza y cuello, Profesor Asistente, Departamento de Ciencias Clínicas, Universidad NeivaHuila. Correo electrónico: adoracu74@hotmail.com. 
Estudios demuestran una relación entre la deficiencia de yodo y el carcinoma folicular, mientras que dietas ricas en yodo incrementan la incidencia relativa del carcinoma papilar ${ }^{(3,4)}$.

La incidencia del cáncer tiroideo en EE.UU. está en aumento ${ }^{(5)}$, ya que representa el $1,5 \%$ de todos los cánceres diagnosticados y se ha incrementado a una tasa de más del $4 \%$ en los últimos 20 años de acuerdo a lo reportado por la sociedad americana de cáncer ${ }^{(2)}$. Aproximadamente 24.000 nuevos pacientes con cáncer tiroideo son diagnosticados en cada año en EE.UU.

En Colombia, de acuerdo con los datos publicados por Globocan en el 2000, la tasa ajustada de incidencia de cáncer de tiroides para hombres fue de 1 por 100.000 habitantes y en mujeres, de 3,7 por 100.000 habitantes $^{(6)}$. De acuerdo con el Instituto Nacional de Cancerología de Colombia, representa la séptima neoplasia más frecuentemente diagnosticada. Para el 2001, se presentaron 30 casos incidentes en hombres y 110 casos en mujeres ${ }^{(7)}$, de los cuales, el $90 \%$ fueron carcinomas bien diferenciados de tipo papilar.

La mortalidad no ha cambiado en los últimos 20 años y la supervivencia a 10 años está estrictamente relacionada con su histología, siendo del 99\% para CTD, $75 \%$ para medular y menos del $5 \%$ para $\mathrm{CA}^{(8)}$. La distribución mundial de los subtipos histológicos, muestra un incremento en el CTD y un descenso en el $\mathrm{CA}^{(9)}$. Esto es probable debido a un mejor diagnóstico y a terapias en enfermedad temprana que previenen la diseminación prolongada y la desdiferenciación.

El primer paso en la terapia de los tumores tiroideos (excepto algunos CA) es la cirugía; la extensión de esta debe ser guiada por el comportamiento biológico e histológico del cáncer. Este artículo revisa las opciones terapéuticas en el manejo quirúrgico del cáncer de tiroides.

\section{MANEJO QUIRÚRGICO}

Las opciones quirúrgicas para el cáncer tiroideo incluyen:

- Cirugía conservativa: hemitiroidectomía más istmectomía.

- Cirugía conservativa más disección central.

- Cirugía radical: tiroidectomía total con o sin linfadenectomía.
La cirugía del cáncer tiroideo debe ser realizada por cirujanos expertos con equipo multidisciplinario. Los cirujanos deben estar familiarizados con la historia natural de los diferentes tumores tiroideos y sus técnicas quirúrgicas, incluyendo tanto tiroidectomía como disección central. Varios estudios demuestran que las complicaciones están asociadas con la experiencia del cirujano(10-12).

\section{CÁNCER TIROIDEO DIFERENCIADO}

Las opciones quirúrgicas incluyen:

- Cirugía conservativa.

- Cirugía conservativa mas disección central.

- Cirugía radical para cáncer avanzado o de alto riesgo.

La extensión de la cirugía siempre ha sido controversial, debido al buen pronóstico de estos pacientes. Hay consenso de manejo para pacientes de alto riesgo, definido por diferentes factores pronósticos como edad, metástasis, extensión y tamaño (AMES); edad, grado, extensión y tamaño (AGES); y tumor primario, adenopatías regionales y metástasis distantes (TNM). En estos pacientes el tratamiento óptimo es la tiroidectomía total ${ }^{(13-17)}$. La extensión de la cirugía en pacientes con bajo riesgo aun permanece en debate. Un estudio prospectivo, randomizado, controlado para evaluar los beneficios o riesgos potenciales de la hemitiroidectomía versus la tiroidectomía es difícil de desarrollar: por el alto número de pacientes requeridos y tiempo de seguimiento que debe realizarse para demostrar alguna diferencia en el pronóstico de ambas conductas.

Los autores que sustentan que la tiroidectomía total es la mejor conducta, afirman las siguientes ventajas ${ }^{(13,18-20)}$ :

- Los focos microscópicos pueden estar presentes en el lóbulo contralateral donde las recurrencias se desarrollan en aproximadamente el 7\% de los pacientes sometidos a cirugía conservativa.

- El número reducido de reintervenciones, disminuye el riesgo de complicaciones.

El yodo radioactivo puede ser usado para detectar y tratar el tejido residual o las metástasis. 
- Los niveles de tiroglobulina sérica, considerada el marcador más sensible de enfermedad recurrente o persistente, son mas fidedignos después de la remoción completa de la glándula tiroides.

- Prevención de la desdiferenciación del tejido tiroideo residual.

- La terapia con supresión hormonal es más sencilla.

Los autores que sustentan la cirugía conservativa para pacientes con bajo riesgo sugieren lo siguiente ${ }^{(17,21,22) \text { : }}$

- La tiroidectomía total está asociada con una mayor tasa de complicaciones.

- Las posibles recurrencias locales pueden ser controladas con cirugía.

- La tasa de recurrencia local en el lecho tiroideo es menor del 5\%.

- La multifocalidad tumoral tiene poca significancia clínica.

- El tejido extrafolicular de la glándula tiroides modula las funciones neuroendocrinas.

- Si la enfermedad tiene un buen pronóstico, la cirugía demolitiva debe ser evitada.

Shaha divide los pacientes en bajo riesgo, alto riesgo y riesgo intermedio, incluyendo en el grupo de riesgo intermedio pacientes menores de 45 años con factores pronósticos desfavorables y mayores de 45 años con factores pronóstico favorables ${ }^{(21)}$. Reportó una supervivencia a 10 años en pacientes con CTD tan alta como $99 \%$ en los pacientes de bajo riesgo, $87 \%$ riesgo intermedio y $57 \%$ los de riesgo alto. Shaha y cols., estudiaron una serie de pacientes de bajo riesgo sometidos a tiroidectomía total vs. tiroidectomía casi total y no encontró diferencia en la supervivencia y recidiva ${ }^{(22)}$. Basado en estos resultados ellos sugieren que la conducta de manejo para pacientes con CDT debe estar basada en los factores pronóstico y en los factores de riesgo individuales (edad, variantes histológicas, exposición previa a radiación, enfermedad familiar y otras). La lobectomía más istmectomía es satisfactoria para el grupo de bajo riesgo; una tiroidectomía total es la mejor opción para el grupo de alto riesgo. La extensión de la tiroidectomía y los posibles tratamien- tos adicionales deben ser realizados de acuerdo a los factores pronóstico en el grupo de riesgo intermedio ${ }^{(21)}$.

La incidencia de metástasis linfáticas en pacientes con CDT es mayor al 50\% y el nivel VI es el principalmente comprometido ${ }^{(2)}$. Por esta razón muchos cirujanos realizan de rutina vaciamiento central en pacientes con cáncer papilar de tiroides, aunque recientes estudios de tipo retrospectivo de cohortes históricas realizados en el Instituto europeo de oncología y en el Instituto Nacional de Cancerología, refutan la conducta del vaciamiento de rutina. El vaciamiento electivo de los niveles II al VI es usualmente realizado únicamente si hay metástasis clínicamente evidentes $^{(2)}$.

No hay consenso alrededor de la utilidad del vaciamiento central en pacientes clínicamente negativos. El carcinoma papilar de tiroides tiende a la extensión a los ganglios linfáticos en los estadios tempranos, incrementando las tasas de recurrencia sin afectar la mortalidad. No hay estudios prospectivos randomizados que prueben que la linfadenectomía profiláctica es superior ${ }^{(23)}$.

La tiroglobulina, la cual puede ser sintetizada tanto por tejido glandular normal como por tejido tumoral, es el único marcador para actividad folicular de tejido tiroideo benigno y maligno. La tiroglobulina es un marcador altamente específico y sensible para CDT cuando los autoanticuerpos están ausentes. Los niveles basales están directamente correlacionados con la cantidad de tejido tiroideo funcionante.

Una variedad de alteraciones genéticas tales como daños inducidos y mutaciones puntuales ocurren en el desarrollo del CDT. Los daños inducidos están ligados con la exposición a la radiación, mientras que el origen de las mutaciones puntuales permanece desconocido.

Los marcadores moleculares de la progresión neoplásica incluyen expresión de la vimentina, fibronectina, osteopontina, RUNX2 y regulación negativa de la E-caderina. La expresión de estos genes es promovida por varios factores de crecimiento con actividad de tirosina quinasa.

Los CDT incluyen cáncer papilar y folicular; el cáncer folicular es el más agresivo y debe ser incluido en el grupo de riesgo intermedio. 


\section{MICROCARCINOMA}

Las opciones de terapia del microcarcinoma incluyen:

- Cirugía conservadora.

- Cirugía conservadora mas vaciamiento central.

- Seguimiento.

El diagnóstico y la terapia del microcarcinoma papilar (CMPT) son debatibles. Es obvio que los grandes carcinomas papilares de tiroides, se originan de pequeños carcinomas papilares, el problema es determinar cuáles de estos crecerán de forma lenta y cuáles podrían ser potencialmente agresivos. El CMPT es definido únicamente por tamaño: un tumor que mide $1 \mathrm{~cm}$ o $\operatorname{menos}^{(24,25)}$.

Existe gran variabilidad en la incidencia de CMPT, que depende de las diferentes características de la población y la exactitud del examen patológico. La incidencia ha sido reportada más del 35\% en Japón, Finlandia y entre inmigrantes japoneses en Hawái. En series de necropsias, el CMPT fue encontrado entre el 3\% al $36 \%$ de pacientes adultos que murieron por enfermedades diferentes a las tiroideas. En series quirúrgicas, la incidencia de CMPT después de la tiroidectomía para enfermedad benigna (bocio nodular y adenoma folicular) es reportada entre el $2 \%$ al $24 \%$. Esto sugiere que la mayoría de estos pueden tener un comportamiento "benigno"(26).

El CMPT no demuestra predilección por sexo comparado con las otras enfermedades tiroideas. Varios factores predisponentes están implicados; los más importantes son la exposición a radiación ionizante y el exceso en la suplementación de yodo. Aunque la multifocalidad en el CMPT es reportada en el $20 \%$ al $46 \%$ de los pacientes, muestra un comportamiento biológico indolente. Sin embargo más del $11 \%$ pueden desarrollar metástasis linfáticas y recurrencias locales.

El CMPT muestra características estructurales citológicas, inmunohistoquímicas y características de comportamiento del cáncer papilar de tiroides; algunos autores reportan una diferencia en la presencia de desmoplasia y en la localización superficial del tumor cercano a la cápsula de la glándula ${ }^{(27)}$.
El CMPT es frecuentemente no detectable clínicamente por su pequeño tamaño y el hecho de que usualmente permanece silente y es descubierto como un hallazgo incidental en la necropsia o en la tiroidectomía por otra enfermedad. La ecografía de cuello en manos expertas puede ser de gran ayuda en el diagnóstico temprano, esta es la técnica imagenológica estándar para la detección de nódulos tiroideos y metástasis linfáticas. La citología aspirativa con aguja fina (ACAF) es el "gold estándar" para el diagnóstico. La ACAF guiada por ecografía tiene una sensibilidad del $60 \%$ a $90 \%$, especificidad del $100 \%$, valor predictivo positivo del $100 \%$ y un valor predictivo negativo del $80 \%$, alcanzando una exactitud del $85 \%(28)$. La ACAF guiada por ecografía puede diagnosticar carcinoma papilar de tiroides en lesiones mayores de 3 a $5 \mathrm{~mm}$.

El CMPT puede desarrollar metástasis linfáticas cuando está asociado con crecimiento acelerado que pueden ser mostrados por la actividad de la proliferación celular, índices apoptoticos y expresión reducida de los supresores de metástasis. Diferentes estudios sugieren factores pronósticos desfavorables, con un incremento en el riesgo de las recurrencias aun en el CMPT (Tabla 1).

Tabla 1. Factores pronósticos desfavorables con incremento en el riesgo de microcarcinoma papilar de tiroides.

Factor pronóstico

Edad mayor de 45 años

Invasion capsular

Tumor no encapsulado

Multifocalidad

Metastasis a ganglios linfáticos

Subexpresión de P27

Expresión incrementada de P53, Bcl-2, c-erbB-2 y P21,ciclina D1, TGF- $\beta 3$

El manejo del CMPT es controversial como el tratamiento del CDT. Algunos autores sugieren que muchos pacientes pueden ser simplemente observados sin ningún tipo de tratamiento o manejados con terapia hormonal supresora ${ }^{(29-}$ 31); brindando cirugía únicamente para los casos con factores pronósticos desfavorables. Estudios posteriores son necesarios para confirmar estas recomendaciones. 


\section{COMPLICACIONES DE LA TIROIDECTOMÍA}

Las complicaciones asociadas con cirugía tiroidea son directamente proporcionales a la extensión de la cirugía e inversamente proporcionales a la experiencia del cirujano. Ocurren con menor frecuencia cuando se realiza una buena técnica quirúrgica con una adecuada compresión de la anatomía quirúrgica. Estas incluyen infección de la herida, seroma, hematoma, lesión nerviosa, hipoparatiroidismo, hipotiroidismo, hemorragia postoperatoria y obstrucción postoperatoria. Otras complicaciones están relacionadas con el vaciamiento ganglionar como la injurian nerviosa y la fístula de quilo (Tabla 2).

\section{COMPLICACIONES ASOCIADAS CON LA HERIDA}

La tiroidectomía está asociada con una baja incidencia de infección y los antibióticos profilácticos de rutina no están indicados. Diferentes estudios han reportado tasas de infección del $0,5 \%{ }^{(32)}$. El seroma, hematoma y la necrosis de los colgajos de piel son poco comunes.

El sangrado intraoperatorio y postoperatorio puede ser venoso o arterial y ocurren en el 1,2 al $1,6 \%$ de los pacientes y pueden conducir a consecuencias fatales debido a la compresión traqueal y la hipoxia ${ }^{(32,33)}$. La mayoría de sangrados ocurren en las primeras 6 horas, pero el $25 \%$ pueden ocurrir entre las 6 a 24 horas postoperatorias. Los drenes no reducen el riesgo de esta complicación. El tratamiento es la reintervención y el drenaje del hematoma.

\section{OBSTRUCCIÓN DE LA VÍA AÉREA}

Es la complicación mayor de la cirugía tiroidea y puede conducir a la muerte. Las razones in- cluyen lesión bilateral del nervio laríngeo recurrente, traqueomalacia, edema subglotico y hemorragia o hematoma postoperatorio ${ }^{(34)}$.

\section{LESIÓN NERVIOSA}

\section{Nervio laríngeo superior}

La lesión de la rama externa del nervio laríngeo superior da como resultado cambios en la voz, ligera ronquera, bajo volumen, voz cansada e incapacidad para cantar notas altas ${ }^{(33)}$. Estas lesiones pueden ser sutiles y no le damos la importancia que requiere este nervio. Se pueden prevenir por la clara identificación de este antes de ligar la arteria tiroidea superior o por la ligadura separadamente de la arteria y la vena tiroidea superior cerca a la glándula tiroides sin la identificación del nervio ${ }^{(35)}$.

\section{Nervio laríngeo recurrente}

Representa la complicación más seria de la tiroidectomía. La incidencia puede variar del $0,3 \%$ al $17 \%$, dependiendo de la habilidad y la experiencia del cirujano ${ }^{(36,37)}$. Hay una mayor incidencia en las reoperaciones. Los factores que pueden influenciar en la incidencia de la complicación incluyen el tipo de procedimiento quirúrgico, las características histológicas y la falla en la identificación del nervio.

Para evitar la lesión, una técnica meticulosa para la identificación del nervio debe ser empleada, teniendo en cuenta que hay una considerable variación anatómica. Las variaciones anatómicas pueden presentarse en cada lado del cuello, esto es porque el opérculo torácico es uno de los tres sitios potenciales donde el nervio esta a riesgo alto, las otras dos áreas de riesgo son en el punto de ligadura de la arteria tiroidea inferior y en el ligamento de Berry ${ }^{(38)}$.

Tabla 2. Principales complicaciones asociadas con cirugía tiroidea.

\begin{tabular}{llll}
\hline Intraoperatoria & Postoperatoria & Metabólica & Asociada a vaciamiento \\
\hline $\begin{array}{l}\text { Lesión nervio } \\
\text { laríngeo recurrente }\end{array}$ & Hemorragia & Hipocalcemia & Lesión nerviosa \\
$\begin{array}{l}\text { Lesión nervio } \\
\text { laríngeo superior }\end{array}$ & Obstrucción de la vía aérea & Fístula de quilo & \\
Lesión combinada & Complicaciones de la herida & & \\
\hline
\end{tabular}


La lesión del nervio laríngeo recurrente puede provocar disfonía, disfagia moderada y compromiso de la vía aérea dependiendo de la extensión de la lesión y del compromiso unilateral o bilateral. La mayoría de cirujanos recomiendan la identificación sistemática del nervio a nivel de la arteria tiroidea inferior donde su recorrido es menos variable ${ }^{(39)}$. Algunos cirujanos recomiendan la monitoria intraoperatoria para la identificación del nervio, pero esto no reemplaza la disección metódica por un cirujano experimentado(33).

\section{HIPOPARATIROIDISMO}

La hipocalcemia postoperatoria puede ser causada por hipoparatiroidismo transitorio o permanente. Frecuentemente se presenta en cáncer tiroideo extenso e invasivo y en la tiroidectomía con vaciamiento central o vaciamiento laterocervical; cuando el tejido paratiroideo es removido y no se reimplanta, ocasionando disrupción de la vasculatura de las glándulas.

La incidencia varia y ha sido reportada del 1,2 al $40 \%{ }^{(32)}$. La mayoría de las personas tiene cuatro glándulas paratiroides localizadas en la cápsula posterolateral de la tiroides, esta localización es variable por que se originan de la tercera y cuarta bolsa faríngea y descienden al cuello.

Cuando las glándulas no pueden ser disecadas de la tiroides con un pedículo vascular adecuado, estas deben ser reimplantadas en el músculo esternocleidomastoideo o en el músculo braquioradial del antebrazo no dominante.

\section{CONCLUSIONES}

El cáncer tiroideo es una neoplasia poco común con variantes histológicas de tumores diferenciados de buen pronóstico a tumores poco diferenciados de pésimo pronóstico. Esta patología debe ser manejada en centros especializados y por cirujanos expertos para minimizar el riesgo de complicaciones. Las opciones quirúrgicas de tratamiento de los tumores diferenciados permanecen divergentes con dos conductas definidas que van de la cirugía conservadora a la cirugía radical. Cada grupo ofrece sus ventajas sin lograrse definir cuál sea la mejor conducta. No existen estudios randomizados por la gran cantidad de pacientes y el largo tiempo de seguimiento requeridos. El microcarcinoma presenta las mismas divergencias en el tratamiento.

\section{REFERENCIAS}

1. Pacini, F., Schlumberger, M., Dralle, H. et al. 2006. European consensus for the management of patients with differentiated thyroid carcinoma of the follicular epithelium. European Journal of Endocrinology 154:787-803.

2. Shaha, AR. 2005. Advances in the management of thyroid cancer. International Journal of Surgery 3:213-220.

3. Deandrea, M., Gallone, G., Veglio, M. et al. 1997. Thyroid cancer histotype changes asobserved in a major general hospital in a21year period. Journal of Endocrinological Investigation 20:52-58.

4. Lind, P., Langsteger, W., Molnar, M. et al. 1998. Epidemiology of thyroid diseases in iodine sufficiency. Thyroid 8:1179-1183.

5. Sosa, J. A. and Udelsman, R. 2006. Total thyroidectomy for differentiated thyroid cancer. Journal of Surgical Oncology 94:701-707.

6. Ferlay, J., Bray, F., Globocan. 2000. Cancer incidence,mortality and prevalence. Worldwide. Version 1.0. Lyon, France. IARC Press. 2001.

7. Pineros, M., Pardo, C., Cantor, L., Hernández, G., Martínez, T. et al. 2002. Instituto Nacional de Cancerología. Registro institucional de cáncer del instituto nacional de cancerología, Empresa social del estado. Resultados principales, año 2001. Revista Colombiana de Cancerología 6:4-49.

8. Hundahl, S.A., Fleming, I.D., Fremgen, A.M. et al. 1998. A National Cancer Data Base report on 53,856 cases of thyroid carcinoma treated in the US. Cancer 83:2638-2648.

9. Cady, B., Rossi, R.L. 1991. Undifferentiated carcinoma and lymphoma of thyroid gland. In: Surgery of the Thyroid and Parathyroid Glands. Cady B, Rossi RL (Eds). WB Saunders, PA, USA 179-186.

10. Sosa, J.A., Bowman, H.M., Tielsch, J.M., Powe, N.R., Gordon, T.A., Udelsman, R. 1998. The importance of surgeon experience for clinical and economic outcomes from thyroidectomy. Annals of Surgery 228(3):320-330.

11. Acun, Z., Cihan, A., Ulukent, S.C. et al. 2004. A randomized prospective study of complications between general surgery residents and attending surgeons in near total thyroidectomies. Surgery Today 34(12):997-1001. 
12. Manolidis, S., Takashima, M., Kirby, M., Scarlet, M. 2001. Thyroid surgery: a comparison of outcomes between experts and surgeons in training. Otolaryngol. Head Neck Surg 125(1):30-33.

13. Dackiw, A., Zeiger, M. 2004. Extent of surgery for differentiated thyroid cancer. Surgery Clinics of North America 84:17-832.

14. Cady, B., Rossi, R. 1988. An expanded view of risk-group definition in differentiated thyroid carcinoma. Surgery 104:947-953.

15. Hay, I.D., Grant, C.S., Taylor, W.F. et al. 1987. Ipsilateral lobectomy versus bilateral lobar resection in papillary thyroid carcinoma: a retrospective analysis of surgical outcome using a novel prognostic scoring system. Surgery 102:1088-1095.

16. Greene, F.L., Page, D.L., Fleming, I.E. et al. (Eds). 2002. American Joint Committee on Cancer Staging Manual (6th Edition). Springer-Verlag, NY, USA 77-87.

17. Udelsman, R., Shaham, A. 2005. Is total thyroidectomy the best possible surgical management for well-differentiated thyroid cancer? Lancet Oncology 6:529-531.

18. Kebebew, E., Clark, O.H. 2000. Differentiated thyroid cancer: "complete" rational approach. World Journal of Surgery 24:942-951.

19. Dackiw, A.P., Clark, O.H. 2003. Bilateral thyroidectomy: indications and technique. In: Surgery of the Thyroid and Parathyroid Glands. Randolph GW (Ed.). WB Saunders, PA, USA 284-291.

20. Jossart, G.H., Clark, O.H. 1994. Welldifferentiated thyroid cancer. Current Problems in Surgery 31:933-1012.

21. Shaha, A.R. 2004. Implications of prognostic factors and risk groups in the management of differentiated thyroid cancer. Laryngoscope 114:393-402.

22. Shaha, A.R., Shan, J.P., Loree, T.R. 1997. Lowrisk differentiated thyroid cancer: the need for selective treatment. Annals of Surgical Oncology 4:328-333.

23. Dionigi, G., Dionigi., R, Bartalena., L, Boni., L, Rovera., F, Villa., F. 2006. Surgery of lymph nodes in papillary thyroid cancer. Expert Review of Anticancer Ther 6(9):1217-1229.

24. Arem, R., Onativa, A., Saliby, AH. et al. 1999. Thyroid microcarcinoma: prevalence, prognosis, and management. Endocrine Practice 5:148-156.

25. Baloch, Z., LiVolsi, A. 2006. Microcarcinoma of the thyroid. Advances in Anatomic Pathology 13(2):69-75.

26. Ramirez, A., Gibelli, B., Tradati, N., Giugliano, G. 2007. Surgical management of thyoridcáncer. Expert Review of Anticancer Ther 7(9): 1023-1214.

27. Salvadori, B., Del Bo, R., Pilotti, S. et al. 1993. 'Occult' papillary carcinoma of the thyroid: a questionable entity. European Journal of Cancer 29A:1817-1820.

28. Bramley, M.D., Harrison, B.J. 1996. Papillary microcarcinoma of the thyroid gland. British Journal of Surgery 83:1674-1683.

29. Harach, H.R., Franssila, K.O., Wasenius, VM. 1985. Occult papillary carcinoma of the thyroid. A "normal" finding in Finland. A systematic autopsy study. Cancer 56:531-538.

30. Ito, Y., Tomoda, C., Uruno, T. et al. 2004. Papillary microcarcinoma of thyroid. How should it be treated? World Journal of Surgery 28:1150-1121.

31. Ito, Y., Miyauchi, A. 2007. A therapeutic strategy for incidentally detected papillary microcarcinoma of the thyroid. Nature Clinical Practice Endocrinology \& Metabolism 3(3):240-248.

32. Bergamaschi, R., Becouarn, G., Ronceray, J., Arnaud, J.P. 1998. Morbidity of thyroid surgery. The American Journal of Surgery 176:71-75.

33. Zarnegar, R., Brunaud, L., Clark, O.H. 2003. Prevention, evaluation and management of complications following thyroidectomy forthyroid carcinoma. Journal of Clinical Endocrinology \& Metabolism 32:483-502.

34. Kennedy, T.L. 2003. Surgical complications ofthyroidectomy. Operative techniques in otolaryngology-head and neck. Surgery 14(2):74-79.

35. Bellantone, R. et al. 2001. Is the identification ofthe external branch of the superior laryngeal nerve mandatory in thyroidoperation? Results of a prospective randomized study. Surgery 130:1055-1059.

36. Jatzko, GR., Lisborg, P.H., Muller, M.G. et al. 1994. Recurrent nerve palsy after thyroid operation-principal nerve identificationand a literature review. Surgery 115:139-144.

37. Al-Suliman, N.N., Ryttov, N.F., Quist, N. et al. 1997. Experience in a specialist thyroid 
surgery unit: a demographic study, surgical complications, and outcome. European Journal of Surgery 163:13-20.

38. Kalky, M.I., Weber, R.S. 1993. Complications ofsurgery of the thyroid and parathyroid glands. Surgical Clinics of North America 73:307-320.
39. Sturniolo, G., D'Alia, C., Tonante, A. et al. 1999. The recurrent laryngeal nerve related to thyroid surgery. The American Journal of Surgery 177:485-188. 